\title{
Parallel transport and layer-resolved thermodynamic measurements in twisted bilayer graphene
}

\author{
G. Piccinini $\odot,{ }^{1,2}$ V. Mišeikis,${ }^{2,3}$ K. Watanabe, ${ }^{4}$ T. Taniguchi, ${ }^{5}$ C. Coletti, ${ }^{2,3,}{ }^{*}$ and S. Pezzini $\odot^{6, \dagger}$ \\ ${ }^{1}$ NEST, Scuola Normale Superiore, Piazza San Silvestro 12, 56127 Pisa, Italy \\ ${ }^{2}$ Center for Nanotechnology Innovation @NEST, Istituto Italiano di Tecnologia, Piazza San Silvestro 12, 56127 Pisa, Italy \\ ${ }^{3}$ Graphene Labs, Istituto Italiano di Tecnologia, Via Morego 30, 16163 Genova, Italy \\ ${ }^{4}$ Research Center for Functional Materials, National Institute for Materials Science, 1-1 Namiki, Tsukuba 305-0044, Japan \\ ${ }^{5}$ International Center for Materials Nanoarchitectonics, National Institute for Materials Science, 1-1 Namiki, Tsukuba 305-0044, Japan \\ ${ }^{6}$ NEST, Istituto Nanoscienze-CNR and Scuola Normale Superiore, Piazza San Silvestro 12, Pisa 56127, Italy
}

(Received 11 June 2021; revised 13 September 2021; accepted 9 November 2021; published 27 December 2021)

\begin{abstract}
We employ dual-gated $30^{\circ}$-twisted bilayer graphene to demonstrate simultaneous ultrahigh mobility and conductivity (up to $40 \mathrm{mS}$ at room temperature), unattainable in a single layer of graphene. We find quantitative agreement with a simple phenomenology of parallel conduction between two pristine graphene sheets, with a gate-controlled carrier distribution. Based on the parallel transport mechanism, we then introduce a method for in situ measurements of the chemical potential of the two layers. This twist-enabled approach, neither requiring a dielectric spacer, nor separate contacting, has the potential to greatly simplify the measurement of thermodynamic quantities in graphene-based systems of high current interest.
\end{abstract}

DOI: 10.1103/PhysRevB.104.L241410

Due to its remarkable charge carrier mobility, graphene is a promising candidate for high-performance devices in applicative fields such as high-frequency electronics and optoelectronics [1-3]. In the case of graphene multilayers, the electrical transport properties strongly depend on the number of layers, as well as on the twist angle between them [4]. In particular, turbostratic multilayer graphene was shown to (potentially) harbor not only a mobility as high as single-layer graphene (SLG), but also larger conductivity $(\sigma)$ [5-9]. This is possible since a finite (typically uncontrolled) twist angle between the layers preserves the gapless linear band dispersion [10], while charge redistribution keeps a relatively low carrier density $(n)$ in the different layers [11]. Moreover, it was suggested that the lowest graphene sheet can efficiently screen the others from detrimental substrate-induced potential fluctuations [12]. However, thus far, experiments addressing the carrier mobility of rotated graphene multilayers have been limited to samples with considerable extrinsic disorder (preventing accurate testing of the transport performance) and a single gate electrode (lacking control on the interlayer charge distribution). We present transport measurements on a dual-gated $30^{\circ}$-twisted bilayer graphene (30TBG) device encapsulated in hexagonal boron nitride (hBN). Thanks to low-energy interlayer decoupling and high device quality, we show that 30TBG replicates the transport properties of two

\footnotetext{
*Corresponding author: camilla.coletti@iit.it

${ }^{\dagger}$ Corresponding author: sergio.pezzini@nano.cnr.it
}

Published by the American Physical Society under the terms of the Creative Commons Attribution 4.0 International license. Further distribution of this work must maintain attribution to the author(s) and the published article's title, journal citation, and DOI. pristine SLG sheets conducting in parallel, including ultrahigh mobility at large carrier concentration.

Furthermore, we show that dual-gated 30TBG enables in situ-or, better said, in device-thermodynamic measurements on the individual graphene layers. Measuring the chemical potential $(\mu)$ as a function of experimental knobs such as $n$ or an external magnetic field $(B)$ can greatly contribute to the understanding of novel two-dimensional systems. To this end, probes such as scanning single-electron transistors have been widely employed on graphene $[13,14]$. Experiments based on capacitance spectroscopy can be performed using either metallic [15,16] or graphitic gates [17]. Kim et al. introduced a double-SLG configuration, allowing reciprocal measurements of $\mu(n, B)$ via dc electrical transport [18]. This technique, however, requires a dielectric spacer (typically several-nanometer-thick hBN) to ensure capacitive coupling between separately contacted graphene sheets [19-21]. Here, we exploit the effective electronic decoupling of large-angle TBG to obtain equivalent information via dc electrical transport in a standard dual-gated device. In particular, by keeping one of the layers charge neutral, it is possible to probe $\mu$ in the other one with a resolution in the $\mathrm{meV}$ range (comparable to hBN-spaced structures [20]), as demonstrated by measurements of the SLG Landau level (LL) energies at $B=250 \mathrm{mT}$.

30TBG is synthesized via chemical vapor deposition (CVD) on $\mathrm{Cu}$, encapsulated in $\mathrm{hBN}$, and processed into a dual-gated device [see sketch in Fig. 1(a) and Supplemental Material [22] for details], using the methods developed in Ref. [23]. The selective growth of 30TBG on $\mathrm{Cu}$ is favored by the interaction between step edges on the $\mathrm{Cu}$ surface and the graphene layers, which triggers the orientation of straight (either zigzag or armchair) graphene edges along the step direction [24]. Transfer of $30 \mathrm{TBG}$ to $\mathrm{SiO}_{2} / \mathrm{Si}$ and $\mathrm{hBN}$ - 
(a)

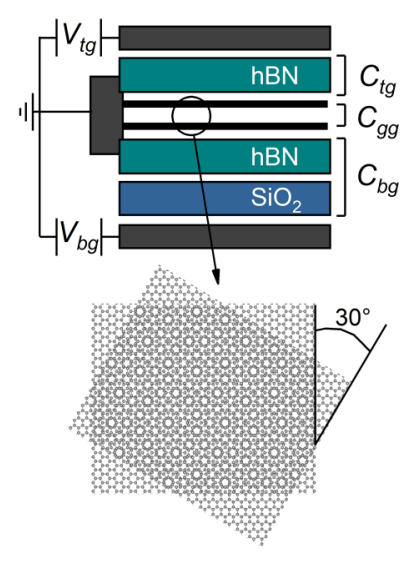

(c)

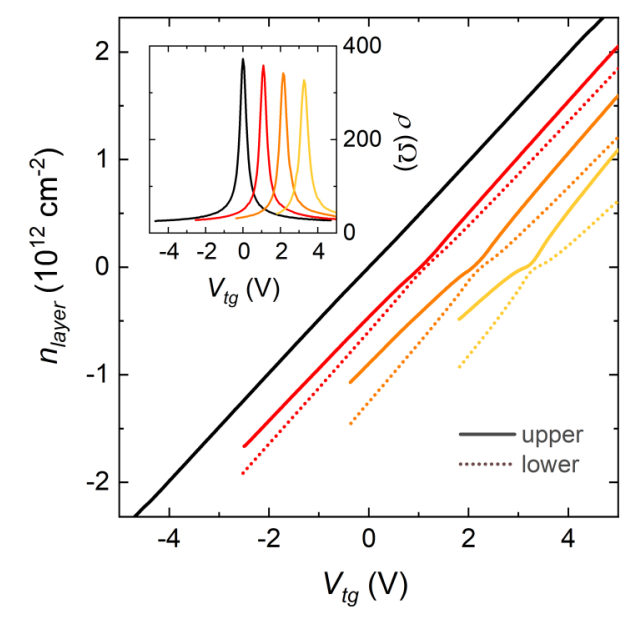

(b)

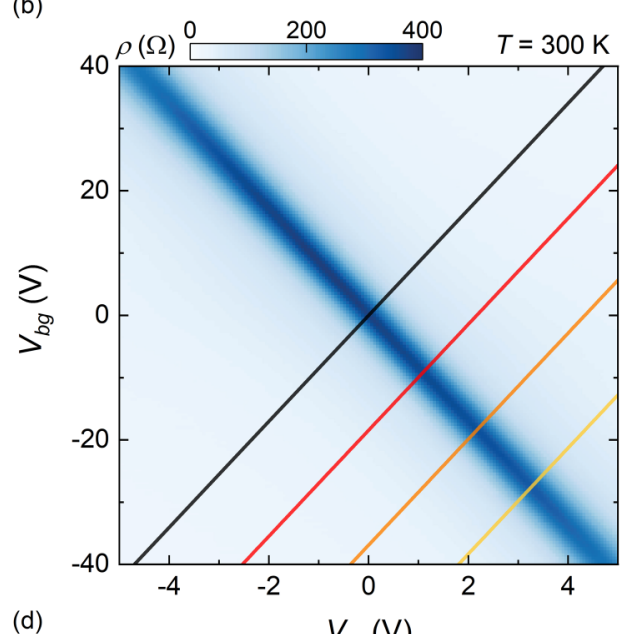

(d)



FIG. 1. Parallel transport and ultrahigh mobility at room temperature. (a) Schematics of the lateral section of the investigated device. CVD-grown $30^{\circ}$-twisted bilayer graphene is encapsulated between $\mathrm{hBN}$ flakes. The potentials $V_{\mathrm{tg}}$ and $V_{\mathrm{bg}}$ are applied to the top and back gate, respectively. The capacitance between the top gate and the upper graphene layer $\left(C_{\mathrm{tg}}\right)$, between the two graphene layers $\left(C_{\mathrm{gg}}\right)$, and between the lower graphene layer and the back gate $\left(C_{\mathrm{bg}}\right)$ are indicated. (b) Longitudinal resistivity of TBG as a function of $V_{\mathrm{tg}}$ and $V_{\mathrm{bg}}$, measured at $T=300 \mathrm{~K}$. The lines indicate different trajectories of the type $V_{\mathrm{tg}} \times C_{\mathrm{tg}}-V_{\mathrm{bg}} \times C_{\mathrm{bg}}=$ const, employed in panels (c) and (d). (c) Computed charge density of the upper (solid lines) and lower (dashed lines) graphene layer along the straight trajectories indicated in panel (b). Inset: longitudinal resistivity peaks as a function of $V_{\mathrm{tg}}$ along the same lines. (d) Mobility of 30TBG as a function of $n_{\text {tot }}$ along the four trajectories. The dashed blue line is the intrinsic phonon-limited mobility of SLG [28], the solid blue line is the intrinsic mobility of two charge-balanced phonon-limited SLG conducting in parallel. Inset: sketch of the four-terminal measurement configuration adopted on the TBG device.

mediated pickup are performed while always keeping $T<$ $170{ }^{\circ} \mathrm{C}$ to avoid possible relaxation of the twist angle. The stability of the $30^{\circ}$-rotated configuration upon hBN encapsulation was confirmed by transmission electron microscope selected area electron diffraction, as reported in Ref. [23]. The assembly technique by Purdie et al. [25] is used to promote interface cleaning and obtain high-quality electronic transport.

The top- and back-gate voltages $\left(V_{\mathrm{tg}}\right.$ and $\left.V_{\mathrm{bg}}\right)$ couple to TBG via $\mathrm{hBN}$ (32 nm thickness, determined by atomic force microscopy) and in-series $\mathrm{SiO}_{2} / \mathrm{hBN}(285 / 40 \mathrm{~nm}$ thickness), respectively, resulting in the capacitance-per-unit-area $C_{\mathrm{tg}}=$ $8.3 \times 10^{-8} \mathrm{~F} / \mathrm{cm}^{2}$ and $C_{\mathrm{bg}}=9.8 \times 10^{-9} \mathrm{~F} / \mathrm{cm}^{2}$. In addition, it is well established that a considerable interlayer capacitance $C_{\mathrm{gg}}=7.5 \times 10^{-6} \mathrm{~F} / \mathrm{cm}^{2}$ must be considered for large-angle TBG [26,27]. The data in Fig. 1(b) show the longitudinal resistivity of $30 \mathrm{TBG}$ measured at room temperature (RT), as a function of $V_{\mathrm{tg}}$ and $V_{\mathrm{bg}}$. The high-resistivity diagonal ( $\rho$ up to $\sim 400 \Omega$ ) corresponds to the global charge neutrality point (CNP) of the sample, while $\rho$ as low as $25 \Omega$ is measured at large carrier concentration. The fact that the global CNP crosses exactly $V_{\mathrm{tg}}=V_{\mathrm{bg}}=0 \mathrm{~V}$ indicates negligible residual doping in the device. As shown by the curves in Fig. 1(c), inset, the resistivity peak becomes wider and shallower upon unbalancing $V_{\mathrm{tg}}$ and $V_{\mathrm{bg}}$, i.e., by increasing the so-called displacement electric field $(D)$. This is caused by a splitting of the CNPs for the two layers [27], although not resolvable at RT due to thermal broadening. To extract quantitative information from the transport data, it is necessary to have knowledge of the individual carrier density in the layers ( $n_{\text {upper }}$ and $n_{\text {lower }}$ ), which can be obtained by electrostatic modeling of the gated TBG system [26,27]; complete details on our procedure are provided in the Supplemental Material [22]. Figure 1(c) shows 
(a)



(c)

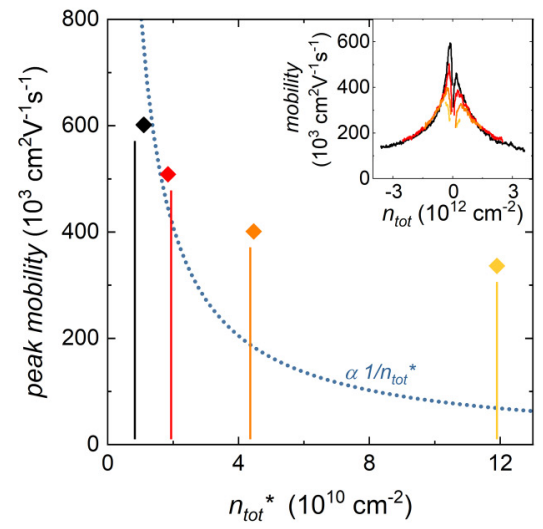

FIG. 2. Low-temperature parallel transport. (a) Longitudinal resistivity as a function of $n_{\text {tot }}$, measured at $T=4.2 \mathrm{~K}$ along the straight trajectories indicated by the lines in the inset. The horizontal markers indicate the maxima of the resistivity peaks. Inset: low $T$ resistivity of $30 \mathrm{TBG}$ as a function of $V_{\mathrm{tg}}$ and $V_{\mathrm{bg}}$. (b) Zoom-in of the longitudinal resistivity as a function of $n_{\mathrm{tot}}$. The blue line is the calculated resistivity of two charge-balanced SLG conducting in parallel, with a carrier mean free path $\left(l_{\mathrm{mfp}}\right)$ equal to the width of our device channel (2.2 $\left.\mu \mathrm{m}\right)$. (c) Measured peak mobility along the different trajectories (maximum values in the inset) as a function of $n_{\text {tot }}{ }^{*}$ (diamonds). $n_{\text {tot }}{ }^{*}$ values obtained from parallel transport simulations (assuming $l_{\mathrm{mfp}}=2.2 \mu \mathrm{m}$ ) are indicated by the vertical lines. The dashed blue line shows the $n_{\mathrm{tot}}{ }^{*}$ dependence of the mobility from Ref. [40], considering parallel transport among two charge-balanced SLG. Inset: mobility of 30TBG as a function of $n_{\text {tot }}$ along the trajectories in panel (a) inset.

the calculated $n_{\text {upper }}$ and $n_{\text {lower }}$ as a function of $V_{\text {tg }}$, along the straight trajectories indicated by lines in Fig. 1(b), which correspond to fixed differences in the gate potentials (weighted with their respective gate capacitance). We note that such trajectories do not coincide with constant values of displacement field (as defined in the electrostatic model in the Supplemental Material [22]), unless at $D=0$ [black line in Fig. 1(b)]. While at $D=0$ the layers are charge balanced (black line, $n_{\text {upper }}=$ $n_{\text {lower }}$ ), increasingly separated and nonlinear $V_{\text {tg }}$ dependencies are observed otherwise. Complete color plots of the $V_{\mathrm{tg}}-V_{\mathrm{bg}}$ dependence of $n_{\text {upper }}$ and $n_{\text {lower }}$ are shown in Fig. S1(a) and S1(b) in the Supplemental Material [22]. Based on such relations, we can calculate the total carrier density in the system $\left(n_{\text {tot }}=n_{\text {upper }}+n_{\text {lower }}\right)$ and use the standard Drude model to convert the experimental $\rho$ data into the mobility $\frac{1}{e\left|n_{\text {tot }}\right| \rho}$ shown in Fig. 1(d). At $n_{\text {tot }}<10^{12} \mathrm{~cm}^{-2}$, we observe a carrier mobility as high as $1.9 \times 10^{5} \mathrm{~cm}^{2} \mathrm{~V}^{-1} \mathrm{~s}^{-1}$, and a weak dependence on the interlayer charge configuration, ascribable to the $D$ induced broadening of the resistivity peak. However, such difference becomes irrelevant at $n_{\text {tot }}>10^{12} \mathrm{~cm}^{-2}$, where the four experimental curves collapse on each other. Here, the RT mobility of 30TBG strikingly surpasses the intrinsic phononlimited behavior of SLG (blue dotted line) [28,29], although falling below the theoretical limit for two parallel-conducting SLG for $n_{\text {tot }}<4 \times 10^{12} \mathrm{~cm}^{-2}$ (blue solid line, calculated assuming $n_{\text {upper }}=n_{\text {lower }}$ and a density-independent resistivity of $52 \Omega$ in each layer [28]). This fact can be reasonably expected based on experiments on hBN-encapsulated SLG, where a RT mobility below the phonon limit is observed at relatively low carrier density $\left(n<2 \times 10^{12} \mathrm{~cm}^{-2}\right)$ [30]. For $n_{\text {tot }}>4 \times$ $10^{12} \mathrm{~cm}^{-2}$, 30TBG perfectly mimics two phonon-limited SLG that simply conduct in parallel, leading to an unprecedented RT mobility at large carrier density $\left(\sim 5 \times 10^{4} \mathrm{~cm}^{2} \mathrm{~V}^{-1} \mathrm{~s}^{-1}\right.$ at $n_{\text {tot }} \sim 5 \times 10^{12} \mathrm{~cm}^{-2}$, corresponding to $\sigma \sim 40 \mathrm{mS}$ ). Such combination might have important applicative implications for high-speed electronics [7,31-33], integrated optoelectronics [34,35], power conversion efficiency in solar cells [36,37], and sensing $[38,39]$.

In Fig. 2, we further investigate the parallel transport mechanism in TBG at low temperature $(T=4.2 \mathrm{~K})$. We again consider several trajectories at fixed gate difference [lines in Fig. 2(a), inset]. However, to avoid a small resistive feature attributable to a contact malfunctioning at $V_{\mathrm{bg}} \sim 0 \mathrm{~V}\left[V_{\mathrm{tg}}-\right.$ independent feature in Fig. 2(a), inset; see also Fig. S2 in the Supplemental Material [22]], we consider half of the $\rho$ curves from the lower left quadrant (for $n_{\text {tot }}<0$ ), half from the upper right one (for $n_{\text {tot }}>0$ ). At low displacement field, the resistivity shows a sharp peak centered at $n_{\text {tot }}=0$, confirming the high quality of the CVD-grown crystals and the low disorder in the hBN-encapsulated device [Fig. 2(a), see also discussion in the Supplemental Material [22]]. With increasing $D$, strong broadening and attenuation [see horizontal markers in Fig. 2(a)] of the peak are observed. Figure 2(b) shows a zoom-in for $\rho \leqslant 60 \Omega$, which allows evaluating the behavior at large $n_{\text {tot }}$. We find that the $30 \mathrm{TBG}$ resistivity perfectly reproduces that of two parallel-conducting graphene layers, in which the carrier mean free path $\left(l_{\mathrm{mfp}}\right)$ is set by the width of the device channel $2.2 \mu \mathrm{m}$, and the conductivity in each SLG is given by $\sigma_{\text {layer }}=2 \frac{e^{2}}{h} l_{\mathrm{mfp}} \sqrt{\pi n_{\text {layer }}}$. This indicates predominant scattering at the device boundaries, consistent with results on hBN-encapsulated SLG [30]. The large- $n_{\text {tot }}$ limit is independent of the interlayer carrier distribution, consistent with the observations at room $T$. However, we find considerable effects on the transport properties at low carrier density. In SLG, the $n^{*}$ parameter-the characteristic density at which $\log (\sigma)$ switches from saturated (electron-hole puddles dominated) to linear-in- $\log (n)$ (single-carrier type)-is determined by long-range disorder and correlates with the inverse of the carrier mobility [40]. In TBG, we find that large values of $n_{\mathrm{tot}}{ }^{*}$ can instead be determined solely by the screening of 



(b)

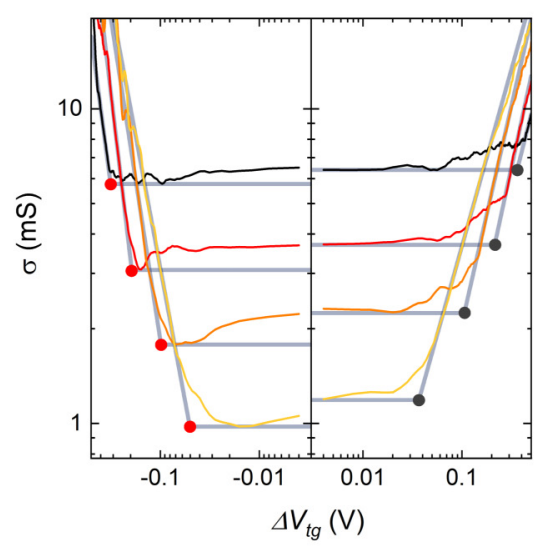

(e)



(c)

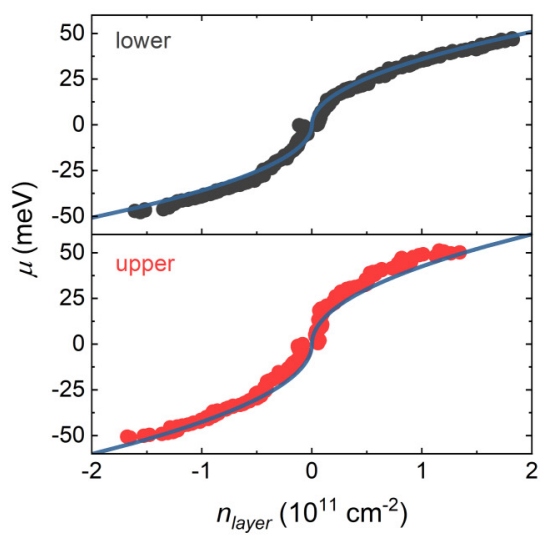

(f)

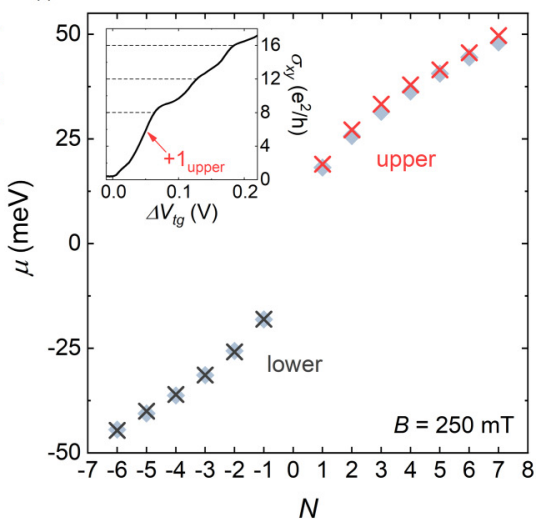

FIG. 3. Layer-resolved thermodynamic measurements in 30TBG. (a) Longitudinal conductivity as a function of $\Delta V_{\mathrm{tg}}$ (top-gate voltage relative to the global CNP) and $V_{\mathrm{bg}}$. The hourglasslike shape in the color plot is due to the split CNPs for the two layers, leading to the four interlayer charge configurations indicated. (b) $\log (\sigma)$ as a function of $\log \left(\Delta V_{\mathrm{tg}}\right)$ along horizontal cuts from panel (a) $\left[V_{\mathrm{bg}}=40 \mathrm{~V}\right.$ to $10 \mathrm{~V}$, see markers in panel (a)]. In the left (right) part, linear fits to $\log (\sigma)$ intersect the minimum conductivity at the CNP of the lower (upper) layer, acting as probe. The corresponding values of gate voltages at the red (black) intersecting points are used to extract $\mu$ and $n$ in the upper (lower) layer, acting as target. (c) Experimentally measured chemical potential as a function of the carrier density for the two graphene layers (black and red circles). The blue lines are fits to the Dirac dispersion, giving the $v_{F}$ values reported in the text. (d) First derivative of the Hall conductivity, as a function of $\Delta V_{\mathrm{tg}}$ and $V_{\mathrm{bg}}$, measured at $B=250 \mathrm{mT}$ and $T=4.2 \mathrm{~K}$. (e) Theoretical LLs positions for the two layers as a function of $\Delta V_{\mathrm{tg}}$ and $V_{\mathrm{bg}}$ [same scales as in panel (d)], computed according to the electrostatic model described in Supplemental Material [22]. (f) Chemical potential of the upper (red crosses) and lower layer (black crosses) as a function of the LL index $N$. The light blue diamonds are the theoretical LL energies at $B=250 \mathrm{mT}$. Inset: Hall conductivity as a function of $\Delta V_{\mathrm{tg}}$, along the dashed line in panel (d). The horizontal lines indicate the quantized plateau values; slight deviations are attributed to residual bulk conduction at low magnetic field. The arrow indicates the crossing point between the lower $N=0$ level and the upper $N=+1$, individuated at the maximum $d \sigma_{\mathrm{xy}} / d V_{\mathrm{tg}}$.

the applied electric field, and do not necessarily correspond to an increased disorder level. The vertical lines in Fig. 2(c) indicate the $n_{\mathrm{tot}}$ * values obtained from parallel transport simulations along the usual trajectories (assuming $l_{\mathrm{mfp}}=2.2 \mu \mathrm{m}$ ), while the experimental points show the measured peak mobility [maximum values in Fig. 2(c), inset] as a function of the extracted $n_{\text {tot }}{ }^{*}$. A peak mobility $\sim 4 \times 10^{5} \mathrm{~cm}^{2} \mathrm{~V}^{-1} \mathrm{~s}^{-1}$ is observed at $n_{\mathrm{tot}}{ }^{*}>10^{11} \mathrm{~cm}^{-2}$ (orange diamond). For comparison [blue dotted line in Fig. 2(c)], two parallel-conducting SLG with a disorder-determined $n_{\text {tot }}{ }^{*}=10^{11} \mathrm{~cm}^{-2}$ (i.e., $5 \times$ $10^{10} \mathrm{~cm}^{-2}$ per layer) are expected to show a mobility $\sim 8 \times$ $10^{4} \mathrm{~cm}^{2} \mathrm{~V}^{-1} \mathrm{~s}^{-1}$ according to the $1 / n^{*}$ dependence for SLG reported in Ref. [40]. Such discrepancy should be taken into careful account, especially in experiments on TBG employing a single gate electrode. The quantitative agreement, both for $\rho$ and $n_{\text {tot }}{ }^{*}$, with simple parallel transport indicates that the interlayer conductivity is negligible with respect to that along the constituent layers [41]. At $30^{\circ}$ twisting, the suppression of interlayer transport might be further favored by the incommensurate stacking configuration [42].

In the following we show the possibility of employing one of the two graphene sheets (the probe) to sense the chemical potential and the carrier concentration in the other one (the target). The key strategy consists in the individuation and tracking of the probe CNP in the parallel transport measurements as a function of the gate potentials. In Fig. 3(a) we plot the low $T$ conductivity as a function of $V_{\mathrm{tg}}$ relative to the global CNP, here defined as $\Delta V_{\mathrm{tg}}=V_{\mathrm{tg}}+V_{\mathrm{bg}} \times C_{\mathrm{bg}} / C_{\mathrm{tg}}$, 
and $V_{\text {bg. }}$. In this zoomed plot, we clearly observe a nonlinear separation between the upper and lower CNPs, resulting from the interlayer capacitive coupling [27]. The CNPs form an hourglasslike shape, separating regions with equal (e-e and $h$ - $h$, high $\sigma)$ and opposite carrier sign ( $e-h$ and $h$-e, low $\sigma)$ on the two layers. The $e-h$ coexistence, expected from calculations of $n_{\text {upper }}$ and $n_{\text {lower }}$ [see Figs. S1(c) and S1(d) in the Supplemental Material [22]], is demonstrated by magnetotransport measurements at $V_{\mathrm{bg}}=-40 \mathrm{~V}$ shown in Fig. S3 in the Supplemental Material [22]. We note that the lower conductivity observed in the $e$ - $h$ configuration is due to the smaller total carrier density and its reduced gate dependence (see Fig. S4 in the Supplemental Material [22]). The position of the probe CNP can be accurately determined by considering $\log (\sigma)$ as a function of $\log \left(\Delta V_{\mathrm{tg}}\right)$ along horizontal cuts from Fig. 3(a), and applying a procedure similar to that commonly used to extract $n^{*}$ in SLG. As shown in Fig. 3(b), crossing one CNP determines a saturation of $\log (\sigma)$, due to the transition from $e-e$ to $h-e$ ( $h-h$ to $h-e$ ) configurations. At the probe $\mathrm{CNP}$, it is possible to calculate both $n$ and $\mu$ in the target layer, using simple relations [20] $n=$ $C_{\mathrm{bg}} V_{\mathrm{bg}} / e+\left(C_{\mathrm{bg}}+C_{\mathrm{gg}}\right) C_{\mathrm{tg}} V_{\mathrm{tg}} /\left(e C_{\mathrm{gg}}\right)$ and $\mu=-e C_{\mathrm{tg}} V_{\mathrm{tg}} / C_{\mathrm{gg}}$ when the upper layer probes the lower one, $n=C_{\mathrm{tg}} V_{\mathrm{tg}} / e+$ $\left(C_{\mathrm{tg}}+C_{\mathrm{gg}}\right) C_{\mathrm{bg}} V_{\mathrm{bg}} /\left(e C_{\mathrm{gg}}\right)$ and $\mu=-e C_{\mathrm{bg}} V_{\mathrm{bg}} / C_{\mathrm{gg}}$ vice versa. Our overall results are shown in Fig. 3(c). The behavior of the experimental points is in agreement with the expected Dirac dispersion $\mu=\operatorname{sgn}(n) \hbar v_{F}(\pi|n|)^{1 / 2}$, with $v_{F}=0.97 \times$ $10^{6} \mathrm{~m} / \mathrm{s}\left(1.15 \times 10^{6} \mathrm{~m} / \mathrm{s}\right)$ estimated for the lower (upper) layer. Since we are unaware of physical mechanisms inducing a different $v_{F}$ in the two layers, we attribute the slight difference to the experimental accuracy in the determination of the CNP position, which is limited by broadening of the CNP by charge fluctuations in each layer $\left(\sim 5 \times 10^{9} \mathrm{~cm}^{-2}\right)$.

By applying a moderate perpendicular magnetic field $[B=$ $250 \mathrm{mT}$, Fig. 3(d)], we observe a series of gate-tunable interlayer quantum Hall states [25]. The LLs from the two layers are bound to transitions between such states, giving large values of $d \sigma_{\mathrm{xy}} / d V_{\mathrm{tg}}$ (first derivative of the Hall conductivity) [23]. The experimental pattern in Fig. 3(d) is well reproduced by the computed LLs positions as a function of the gate potentials, reported in Fig. 3(e), where $n_{\text {upper(lower) }}=e B / h \times 4 N$ ( $N=0, \pm 1, \pm 2, \ldots$ and 4 accounts for the spin and valley degeneracy in each layer). However, we note that this calculation does not consider the quantization of the energy spectrum, and therefore cannot quantitatively match all the details in the experimental data. In presence of $B$, the thermodynamic measurements can rely on the crossings between the zeroenergy LL of the probe layer (pinned at the probe CNP) and the finite-energy LLs of the target layer. As shown in the inset of Fig. 3(f), each of these crossings results in an eightfold quantized step in $\sigma_{x y}$. Considering the position of the corresponding maximum in $d \sigma_{\mathrm{xy}} / d V_{\mathrm{tg}}$ [red arrow in Fig. 3(f) inset] and using the same relations as in zero field, we can obtain the chemical potential at the target LLs, shown in Fig. 3(f). Due to a general lower quality of the $d \sigma_{\mathrm{xy}} / d V_{\mathrm{tg}}$ data for $V_{\mathrm{bg}}>0$, here we employ only the crossings at $V_{\mathrm{bg}}<0$, providing $\mu$ for the upper (lower) layer for $N>0(N<0)$. We compare these data with the LL energies $E(N)=\operatorname{sgn}(N) v_{F}(2 e \hbar B|N|)^{1 / 2}$ [light blue diamonds in Fig. 3(f), $v_{\mathrm{F}}=10^{6} \mathrm{~m} / \mathrm{s}$ ]. The measurement correctly captures the $|N|^{1 / 2}$ dependence and the large scale of $E( \pm 1)$ with respect to the energy separation of the higher-index LLs, both hallmarks of the Dirac dispersion. The standard deviation between the theoretical LL energies and the experimental $\mu$ values is $1.4 \mathrm{meV}$, which sets our current resolution for thermodynamic measurements in magnetic fields.

In principle, our approach can be used to obtain high-resolution thermodynamic information on an arbitrary graphene-based system (for instance, magic-angle TBG as in Ref. [20]), simply by stacking it on top of SLG and imposing a large relative twisting. We stress that the $30^{\circ}$ rotation is not a strict requirement: smaller twist angles could be equivalently employed, as long as the interlayer decoupling is preserved $[26,27]$. While in the case presented here of SLG probing SLG, the value of the interlayer capacitance $C_{g g}$ is well established, this might vary significantly when considering a different target system. However, given the reciprocity of the technique, $C_{g g}$ can be obtained by measuring $\mu(n)$ of the probe SLG via tracking the target CNP, initializing a reasonable starting value of interlayer capacitance (e.g., that of largeangle TBG) and iterating the procedure until convergence to the SLG Dirac dependence. We note that both the parallel transport mechanism and the thermodynamic measurement scheme could be extended to other van der Waals systems where interlayer decoupling is obtained from large-angle twisting, such as twisted transition metal dichalcogenides.

In summary, we have shown that it is possible to achieve simultaneous ultrahigh mobility and conductivity in 30TBG, based on parallel conduction and gate-controlled carrier distribution. In addition, we have exploited the low-energy electronic decoupling of 30TBG to introduce a technique for layer-sensitive thermodynamic measurements using a standard device structure and measurement configuration.

Note added. Most recent work on double-gated $30^{\circ}$ twisted [43] and large-angle-twisted [44] bilayer graphene shows low-temperature transport features consistent with our findings.

We thank F. Rossella for technical support during the low temperature experiments. Growth of hexagonal boron nitride crystals was supported by the Elemental Strategy Initiative conducted by the MEXT, Japan, Grant No. JPMXP0112101001, JSPS KAKENHI Grant No. JP20H00354, and the CREST (Grant No. JPMJCR15F3), JST. The research leading to these results has received funding from the European Union's Horizon 2020 research and innovation program under Grant Agreements No. 785219-Graphene Core2 and No. 881603-Graphene Core3.
[1] Y. M. Lin, K. A. Jenkins, V. G. Alberto, J. P. Small, D. B. Farmer, and P. Avouris, Operation of graphene transistors at gigahertz frequencies, Nano Lett. 9, 422 (2009).
[2] C. H. Yeh, P. Y. Teng, Y. C. Chiu, W. T. Hsiao, S. S. H. Hsu, and P. W. Chiu, Gigahertz fieldeffect transistors with cmos-compatible transfer-free 
graphene, ACS Appl. Mater. Interfaces 11, 6336 (2019).

[3] V. Sorianello, M. Midrio, G. Contestabile, I. Asselberghs, J. Van Campenhout, C. Huyghebaert, I. Goykhman, A. Ott, A. Ferrari, and M. Romagnoli, Graphene-silicon phase modulators with gigahertz bandwidth, Nat. Photonics 12, 40 (2018).

[4] A. V. Rozhkov, A. O. Sboychakov, A. L. Rakhmanov, and F. Nori, Electronic properties of graphene-based bilayer systems, Phys. Rep. 648, 1 (2016).

[5] J. Hass, J. E. Millán-Otoya, M. Sprinkle, N. Sharma, W. A. de Heer, C. Berger, P. N. First, L. Magaud, and E. H. Conrad, Why Multilayer Graphene on 4H-SiC(0001) Behaves Like a Single Sheet of Graphene, Phys. Rev. Lett. 100, 125504 (2008).

[6] R. Negishi, Y. Ohno, K. Maehashi, K. Matsumoto, and Y. Kobayashi, Carrier transport properties of the field effect transistors with graphene channel prepared by chemical vapor deposition, Jpn. J. Appl. Phys. 51, 06FD03 (2012).

[7] K. Uemura, T. Ikuta, and K. Maehashi, Turbostratic stacked CVD graphene for high-performance devices, Jpn. J. Appl. Phys. 57, 030311 (2018).

[8] X. Wu, Y. Chuang, A. Contino, B. Sorée, S. Brems, Z. Tokei, M. Heyns, C. Huyghebaert, and I. Asselberghs, Boosting carrier mobility of synthetic few layer graphene on $\mathrm{SiO}_{2}$ by interlayer rotation and decoupling, Adv. Mater. Interfaces 5, 1800454 (2018).

[9] M. S. Kim, M. Kim, S. Son, S.-Y. Cho, S. Lee, D.-K. Won, J. Ryu, I. Baes, H.-M. Kim, and K.-B. Kim, Sheet resistance analysis of interface-engineered multilayer graphene: Mobility versus sheet carrier concentration, ACS Appl. Mater. Interfaces 12, 30932 (2020).

[10] S. Shallcross, S. Sharma, E. Kandelaki, and O. A. Pankratov, Electronic structure of turbostratic graphene, Phys. Rev. B 81, 165105 (2010).

[11] Y. Gao and S. Okada, Carrier distribution control in bilayer graphene under a perpendicular electric field by interlayer stacking arrangements, Appl. Phys. Express 14, 035001 (2021).

[12] C. P. Lu, M. Rodriguez-Vega, G. Li, A. Luican-Mayer, K. Watanabe, T. Taniguchi, E. Rossi, and E. Y. Andrei, Local, global, and nonlinear screening in twisted double-layer graphene, Proc. Natl. Acad. Sci. USA 113, 6623 (2016).

[13] J. Martin, N. Akerman, G. Ulbricht, T. Lohmann, J. Smet, K. Klitzing, and A. Yacoby, Observation of electron-hole puddles in graphene using a scanning single-electron transistor, Nat. Phys. 4, 144 (2008).

[14] J. Martin, B. E. Feldman, R. T. Weitz, M. T. Allen, and A. Yacoby, Local Compressibility Measurements of Correlated States in Suspended Bilayer Graphene, Phys. Rev. Lett. 105, 256806 (2010).

[15] A. F. Young, C. R. Dean, I. Meric, S. Sorgenfrei, H. Ren, K. Watanabe, T. Taniguchi, J. Hone, K. L. Shepard, and P. Kim, Electronic compressibility of layer-polarized bilayer graphene, Phys. Rev. B 85, 235458 (2012).

[16] E. A. Henriksen and J. P. Eisenstein, Measurement of the electronic compressibility of bilayer graphene, Phys. Rev. B 82, 041412(R) (2010).

[17] A. A. Zibrov, C. Kometter, H. Zhou, E. M. Spanton, T. Taniguchi, K. Watanabe, M. P. Zaletel, and A. F. Young, Tunable interacting composite fermion phases in a half-filled bilayer-graphene Landau level, Nature (London) 549, 360 (2017).
[18] S. Kim, I. Jo, D. C. Dillen, D. A. Ferrer, B. Fallahazad, Z. Yao, S. K. Banerjee, and E. Tutuc, Direct Measurement of the Fermi Energy in Graphene Using a Double-Layer Heterostructure, Phys. Rev. Lett. 108, 116404 (2012).

[19] K. Lee, B. Fallahazad, J. Xue, D. C. Dillen, K. Kim, T. Taniguchi, K. Watanabe, and E. Tutuc, Chemical potential and quantum Hall ferromagnetism in bilayer graphene, Science 345, 6192 (2014).

[20] J. M. Park, Y. Cao, K. Watanabe, T. Taniguchi, and P. JarilloHerrero, Flavour hund's coupling, correlated chern gaps, and diffusivity in moiré flat bands, Nature (London) 592, 43 (2021).

[21] F. Yang, A. A. Zibrov, R. Bai, T. Taniguchi, K. Watanabe, M. P. Zaletel, and A. F. Young, Experimental Determination of the Energy per Particle in Partially Filled Landau Levels, Phys. Rev. Lett. 126, 156802 (2021).

[22] See Supplemental Material at http://link.aps.org/supplemental/ 10.1103/PhysRevB.104.L241410 for details on device fabrication and measurement setup, description of the electrostatic model, additional low-temperature resistivity curves, discussion on extrinsic disorder in CVD-grown 30TBG, additional data on $e-h$ coexistence at high displacement field, and discussion on gate-dependent total carrier density in the $e$ - $h$ configuration.

[23] S. Pezzini, V. Miseikis, G. Piccinini, S. Forti, S. Pace, R. Engelke, F. Rossella, K. Watanabe, T. Taniguchi, P. Kim, and C. Coletti, $30^{\circ}$-Twisted bilayer graphene quasicrystals from chemical vapor deposition, Nano Lett. 20, 3313 (2020).

[24] Z. Yan, Y. Liu, Long Ju, Z. Peng, J. Lin, G. Wang, H. Zhou, C. Xiang, E. L. G. Samuel, C. Kittrell, V. I. Artyukhov, F. Wang, B. I. Yakobson, and J. M. Tour, Large hexagonal bi-and trilayer graphene single crystals with varied interlayer rotations, Angew. Chem. 53, 1591 (2014).

[25] D. G. Purdie, N. M. Pugno, T. Taniguchi, K. Watanabe, A. C. Ferrari, and A. Lombardo, Cleaning interfaces in layered materials heterostructures, Nat. Commun. 9, 5387 (2018).

[26] J. D. Sanchez-Yamagishi, T. Taychatanapat, K. Watanabe, T. Taniguchi, A. Yacoby, and P. Jarillo-Herrero, Quantum Hall Effect, Screening, and Layer-Polarized Insulating States in Twisted Bilayer Graphene, Phys. Rev. Lett. 108, 076601 (2012).

[27] P. Rickhaus, M.-H. Liu, M. Kurpas, A. Kurzmann, Y. Lee, H. Overweg, M. Eich, R. Pisoni, T. Taniguchi, K. Watanabe, K. Richter, K. Ensslin, and T. Ihn, The electronic thickness of graphene, Sci. Adv. 6, eaay8409 (2020).

[28] E. H. Hwang and S. Das Sarma, Acoustic phonon scattering limited carrier mobility in two-dimensional extrinsic graphene, Phys. Rev. B 77, 115449 (2008).

[29] C. H. Park, N. Bonini, T. Sohier, G. Samsonidze, B. Kozinsky, M. Calandra, F. Mauri, and N. Marzari, Electron-phonon interactions and the intrinsic electrical resistivity of graphene, Nano Lett. 14, 1113 (2014).

[30] L. Wang, I. Meric, P. Y. Huang, Q. Gao, Y. Gao, H. Tran, T. Taniguchi, K. Watanabe, L. M. Campos, D. A. Muller, J. Guo, P. Kim, J. Hone, K. L. Shepard, and C. R. Dean, One-dimensional electrical contact to a two-dimensional material, Science $\mathbf{3 4 2}$, 614 (2013).

[31] Y. M. Lin, C. Dimitrakopoulos, K. A. Jenkins, D. B. Farmer, H.-Y. Chiu, A. Grill, and Ph. Avouris, 100-GHz transistors from wafer-scale epitaxial graphene, Science 327, 662 (2010). 
[32] Y. Lin, H. Chiu, K. A. Jenkins, D. B. Farmer, P. Avouris, and A. Valdes-Garcia, Dual-gate graphene FETs with $f_{T}$ of $50 \mathrm{GHz}$, IEEE Electron Device Lett. 31, 68 (2009).

[33] L. Viti, D. G. Purdie, A. Lombardo, A. C. Ferrari, and M. S. Vitiello, HBN-encapsulated, graphene-based, roomtemperature terahertz receivers, with high speed and low noise, Nano Lett. 20, 3169 (2020).

[34] F. Bonaccorso, Z. Sun, T. Hasan, and A. C. Ferrari, Graphene photonics and optoelectronics, Nat. Photonics 4, 611 (2010).

[35] M. A. Giambra, V. Mišeikis, S. Pezzini, S. Marconi, A. Montanaro, F. Fabbri, V. Sorianello, A. C. Ferrari, C. Coletti, and M. Romagnoli, Wafer-Scale Integration of Graphene-Based Photonic Devices, ACS Nano 15, 3171 (2021).

[36] Y. Wang, S. W. Tong, X. F. Xu, B. Özyilmaz, and K. P. Loh, Interface engineering of layer-by-layer stacked graphene anodes for high-performance organic solar cells, Adv. Mater. 23, 1514 (2011).

[37] X. Kong, L. Zhang, B. Liu, H. Gao, Y. Zhang, H. Yan, and X. Song, Graphene/Si Schottky solar cells: A review of recent advances and prospects, RSC Adv. 9, 863 (2019).

[38] Y. Ohno, K. Maehashi, Y. Yamashiro, and K. Matsumoto, Electrolyte-gated graphene field-effect transistors for detecting pH and protein adsorption, Nano Lett. 9, 3318 (2009).
[39] J. Dauber, A. A. Sagade, M. Oellers, K. Watanabe, T. Taniguchi, D. Neumaier, and C. Stampfer, Ultra-sensitive Hall sensors based on graphene encapsulated in hexagonal boron nitride, Appl. Phys. Lett. 106, 193501 (2015).

[40] N. J. G. Couto, D. Costanzo, S. Engels, D.-K. Ki, K. Watanabe, T. Taniguchi, C. Stampfer, F. Guinea, and A. F. Morpurgo, Random Strain Fluctuations as Dominant Disorder Source for High-Quality On-Substrate Graphene Devices, Phys. Rev. X 4, 041019 (2014).

[41] Y. Kim, H. Yun, S.-G. Nam, M. Son, D. S. Lee, D. C. Kim, S. Seo, H. C. Choi, H.-J. Lee, S. W. Lee, and J. S. Kim, Breakdown of the Interlayer Coherence in Twisted Bilayer Graphene, Phys. Rev. Lett. 110, 096602 (2013).

[42] R. Bistritzer and A. H. MacDonald, Transport between twisted graphene layers, Phys. Rev. B 81, 245412 (2010).

[43] S. Slizovskiy, A. Garcia-Ruiz, A. I. Berdyugin, N. Xin, T. Taniguchi, K. Watanabe, A. K. Geim, N. D. Drummond, and I V. Fal'ko, Out-of-Plane Dielectric Susceptibility of Graphene in Twistronic and Bernal Bilayers, Nano Lett. 21, 6678 (2021).

[44] A. Mrenca-Kolasinska, P. Rickhaus, G. Zheng, K. Richter, T. Ihn, K. Ensslin, and M-H. Liu, Quantum capacitive coupling between large-angle twisted graphene layers, arXiv:2110.00907. 